Rogério de Moraes Mattos

\title{
Eficácia da associação de tadalafila e fluoxetina de liberação lenta no tratamento da ejaculação precoce
}

Tese apresentada à Faculdade de Medicina da Universidade de São Paulo para obtenção do título de Doutor em Ciências

Área de concentração: Urologia

Orientador: Prof. Dr. Antonio Marmo Lucon

São Paulo

2005 
RESUMO:

Introdução e objetivos: A ejaculação precoce é uma forma de disfunção sexual presente em 25,8\% dos homens brasileiros. O objetivo do presente estudo é avaliar se a associação de tadalafila, um inibidor da fosfodiesterase-5 e fluoxetina, um inibidor da recaptação da serotonina em uma apresentação de liberação lenta, ambos tomados uma vez por semana, pode prolongar o tempo de latência da ejaculação em homens com ejaculação precoce.

Pacientes e Métodos: Sessenta pacientes com ejaculação precoce e sem disfunção erétil foram avaliados. A idade média foi 45,5 anos de idade (desvio padrão +/- 9,6). O tempo médio de ejaculação antes do início do tratamento era 51,3 segundos (desvio padrão +/- 23 segundos) e não foi estatisticamente significativo entre os grupos $(\mathrm{p}=0,805)$. Foram distribuídos de forma aleatória e duplo-cega em 4 grupos, conforme a medicação recebida: (1) fluoxetina 90 mg e placebo, (2) tadalafila 20 mg e fluoxetina $90 \mathrm{mg}$, (3) tadalafila $20 \mathrm{mg}$ e placebo, e (4) placebo com placebo. Antes de iniciar qualquer medicamento, os pacientes anotaram o tempo de latência para ejaculação com um mesmo cronômetro uma vez por semana, durante 3 semanas. À partir do início do uso dos medicamentos, os pacientes cronometraram o tempo em nove ocasiões, uma vez por semana. Foi usado fluoxetina $90 \mathrm{mg}$ ou placebo semanalmente e tadalafila 20 mg ou placebo em um intervalo de até 36 horas da presumida relação sexual com parceira heterossexual regular. Os pacientes foram prospectivamente seguidos a cada 3 semanas durante 12 semanas. 
Resultados: A comparação dos grupos com análise de variância (ANOVA) unidirecional demonstrou diferença estatisticamente significativa no tempo de ejaculação após tratamento $(\mathrm{p}<0,001)$. O maior aumento em relação ao tempo basal foi observado no grupo que associou tadalafila $20 \mathrm{mg}$ com fluoxetina $90 \mathrm{mg}$ semanalmente $(\mathrm{p}<0,001)$. Reações adversas foram observadas, tendo sido toleradas e equivalentes entre os grupos usando princípio ativo.

Conclusão: Tadalafila 20 mg utilizada em um período de 36 horas de atividade sexual associado com fluoxetina 90 mg de liberação lenta usada semanalmente, significativamente aumentou o tempo de latência de ejaculação em homens com ejaculação precoce, quando comparados com cada droga usada isoladamente, beneficiando esses pacientes sem a necessidade do uso diário de medicamentos. 
SUMMARY:

Introduction and Objectives: Premature ejaculation is a sexual disorder present in $25.8 \%$ of brazilian men. The aim of the present study is to evaluate if the association of tadalafil, a phosphodiesterase-5 inhibitor and fluoxetine, a selective serotonin reuptake inhibitor in a slow release presentation, both taken once a week, can prolong the intravaginal ejaculatory latency time (IELT) in men with premature ejaculation.

$\underline{\text { Methods: }}$ Sixty patients with premature ejaculation and no erectile dysfunction were enrolled in the protocol. Mean age was 45.5 years (range 24 - 64 years, standard deviation +/- 9.6). They were randomly assigned in a double-blind manner into 4 groups to use the medications: (1) fluoxetine $90 \mathrm{mg}$ and placebo, (2) tadalafil $20 \mathrm{mg}$ and fluoxetine $90 \mathrm{mg}$, (3) tadalafil $20 \mathrm{mg}$ and placebo, and (4) two different placebo capsules. Before starting any medication, each individual timed the IELT with a given stopwatch in 3 different days, and likewise weekly during the treatment period. Mean IELT before starting treatment was 51.3 seconds (sd: +/- 23 seconds), and was not different between groups ( $\mathrm{p}=0.805$ ). They took fluoxetine $90 \mathrm{mg}$ or placebo once a week, and tadalafil $20 \mathrm{mg}$ or placebo in a 36-hour frame of intended sexual intercourse with a regular heterosexual partner. Patients were prospectively followed every 3 weeks during a 12-week interval. 
Results: Comparison between groups with oneway ANOVA demonstrated a statistically significant difference in post-treatment IELT $(\mathrm{p}<0.001)$. The greatest increase in time from baseline IELT was observed in patients in the tadalafil plus fluoxetine group $(\mathrm{p}<0.001)$. Side effects were observed and tolerated, being equivalent in groups using active drugs.

Conclusion: Tadalafil 20 mg taken in a 36-hour window for sexual intercourse associated with fluoxetine $90 \mathrm{mg}$ in a slow release form taken weekly, significantly increased the intravaginal ejaculatory latency time from baseline in men with premature ejaculation, when compared to either drug taken solely, benefiting patients without the need to be medicated on a daily basis. 


\section{INTRODUÇÃO:}

A disfunção ejaculatória é uma das alterações sexuais mais comum em homens, com variações entre ejaculação precoce, incapacidade de ejacular, ejaculação inibida e ejaculação retrógrada (1).

As primeiras definições propostas para ejaculação precoce levavam em consideração o tempo decorrido entre a penetração e a ejaculação. Períodos entre 30 segundos e 5 minutos foram arbitrariamente sugeridos, sem atingirem um consenso (2). Cooper (3) propôs o tempo inferior à dois minutos diagnosticando a doença. Outra definição valorizava os movimentos pélvicos necessários para se atingir o orgasmo, sendo caracterizada precoce quando a ejaculação ocorria em poucos movimentos $(4,5)$. Masters e Johnson (6) propuseram uma das primeiras definições em que se focalizava a incapacidade do homem retardar a ejaculação o suficiente para permitir que a parceira tivesse orgasmo em 50\% das relações, assumindo que a ejaculação precoce seria a única causa de anorgasmia feminina. Kaplan (7) foi o primeiro a propor que a ejaculação precoce seria uma falência do controle voluntário sobre o tempo de latência da ejaculação, conceito sobre o qual a definição corrente é baseada. O Manual da Associação Americana de Psiquiatria, $4^{\mathrm{a}}$ edição (8) define a ejaculação precoce como a ejaculação persistente ou recorrente com estimulação sexual mínima antes, durante ou logo após a penetração, e antes que o indivíduo o deseje, que cause acentuado sofrimento ou dificuldade interpessoal e não seja por efeito de uma substância (por exemplo: abstinência de opióides). Esta definição foi 
classificada em dois tipos: primária, que ocorre desde o início da vida sexual e se mantém assim, e secundária, que seria uma forma adquirida (9).

A prevalência da doença nos Estados Unidos é de 21\% em homens entre 18 e 59 anos de idade (10). Também há relatos de prevalência variando de menos de 5\% (11) até mais que 30\% $(12,13,14)$. A ejaculação precoce está presente em 25,8\% dos homens brasileiros pesquisados (15). Na avaliação brasileira, a distribuição da doença por faixas etárias foi similar desde os 18 anos até acima de 61 anos, era primária em 70,5\% dos homens, ocorria logo após a penetração em 45,7\% e também na masturbação em 38,2\%. A frequência do distúrbio nos 13 estados brasileiros pesquisados foi estatisticamente semelhante, além de haver disfunção erétil associada, em 13,6\% dos casos (15).

A etiologia exata da ejaculação precoce ainda é desconhecida. Causas psicológicas, comportamentais e orgânicas foram sugeridas (16).

O tratamento da ejaculação precoce é proposto após anamnese médica e sexual junto com exame físico de cada paciente (17). A anotação do tempo decorrido entre a penetração vaginal e a ejaculação não é um fator para o diagnóstico, mas é utilizado como um critério objetivo nos estudos clínicos.

Durante muito tempo a etiologia da ejaculação precoce ficou associada somente aos quadros de ansiedade e depressão. Os primeiros tratamentos da ejaculação precoce ficaram concentrados na esfera da psicologia, particularmente na 
terapia comportamental. O tempo para esses tratamentos mostrarem resultados eram longos (18). Por outro lado, surgiram tratamentos medicamentosos, como a aplicação tópica de cremes no pênis, e o uso oral de antidepressivos tricíclicos e antidepressivos inibidores de recaptação de serotonina. O uso de cremes tópicos foi descrito retardando a ejaculação em até 89,2\% dos casos (19). O retardo da ejaculação com uso de psicotrópicos de uso contínuo é variável e ocorre em 53\% com antidepressivos tricíclicos e entre 25\% e 42\% com antidepressivos inibidores da recaptação da serotonina (20). Os inibidores da 5 fosfodiesterase também apresentaram efeitos no prolongamento do tempo até a ejaculação (21).

Dois estudos experimentais associaram os inibidores de 5 fosfodiesterase e os antidepressivos inibidores de recaptação de serotonina $(22,23)$ com resultados melhores do que o uso isolado de cada um destes medicamentos, mas ainda não foi definida qual seria a melhor associação entre as drogas disponíveis atualmente e nem qual seria a melhor posologia destas drogas.

A farmacocinética dessas drogas e o comportamento sexual do brasileiro na freqüência de relações sexuais, pode sugerir que as formas de liberação lenta dos medicamentos poderia trazer benefícios para os homens com ejaculação precoce. 


\section{REVISÃO DE LITERATURA:}

Em 1887, Gross (24) publicou o primeiro caso de ejaculação precoce descrito. Quatorze anos depois em outro artigo, von Kraft-Ebing (25) foi o seguinte a abordar o mesmo assunto. Além da raridade e do interesse histórico dessas publicações, a ejaculação precoce era vista naquela época como uma anormalidade física e não como um distúrbio psicológico.

Em 1917, Abraham (26) publicou artigo conceituando a ejaculatio praecox como sintoma de uma neurose causada por conflitos inconscientes. Ele propôs que o tratamento deveria ser com a psicanálise clássica. Um artigo de 1943 de Shapiro (27) sugeria que a ejaculação precoce não era um distúrbio puramente psicológico ou puramente somático, mas psicossomático e causado por uma combinação de personalidade ansiosa junto com um sistema ejaculatório frágil. Desta forma classificou-a em dois tipos, o tipo A eram homens que evoluíam para a disfunção erétil e o tipo B eram homens com ejaculação precoce desde a primeira relação sexual. Após alguns anos, essa terminologia foi atualizada para as classificações primária e secundária de ejaculação precoce, que são usadas até hoje (9).

Durante a relação sexual do homem ocorre a seguinte sequência de eventos: desejo, excitação, orgasmo e resolução. A emissão do sêmen, o fechamento do colo vesical e a ejaculação são eventos reflexos. A propagação da sensibilidade da glande 
é transmitida através do nervo dorsal do pênis para a porção sensorial do nervo pudendo em direção ao trato sensorial superior. (Figura 1).

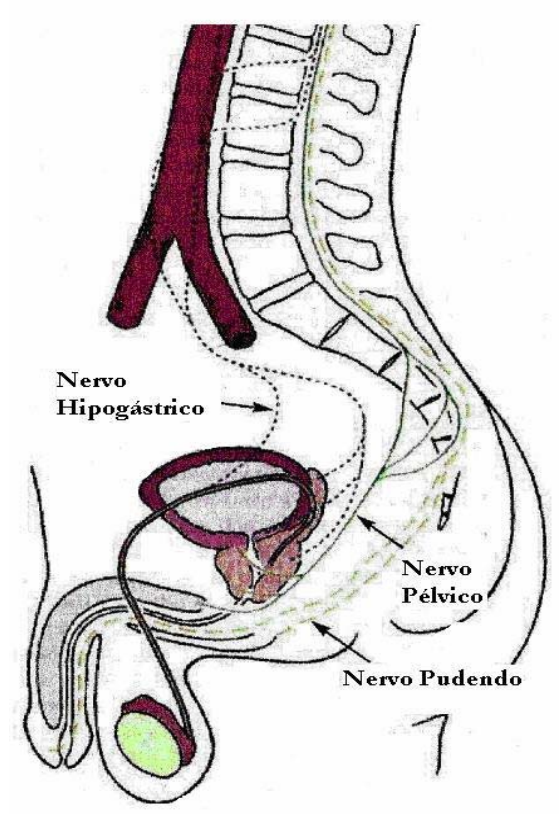

Figura 1: Inervação genital masculina referente à ejaculação.

A emissão e o fechamento do colo vesical são controlados pelo sistema nervoso simpático, que atua por intermédio dos receptores adrenérgicos. O impulso motor simpático eferente é mediado pelas células na coluna lateral da substância cinzenta na medula torácica e medula lombar superior. Quando o sistema simpático é ativado, as vesículas seminais e os deferentes são contraídos, liberando seu fluido na uretra posterior pelos dutos ejaculatórios.

A ejaculação é um reflexo estimulado pela distensão da uretra posterior em resposta à emissão seminal (28). Simultaneamente, o colo vesical se fecha vigorosamente, impedindo o fluido de retroceder para a bexiga. O esfíncter urinário 
interno, em controle somático, também se contrai prevenindo o fluido de sair da fossa prostática. Após a emissão, o centro do reflexo ejaculatório envia um sinal à musculatura que envolve a região peri-uretral resultando em contrações ritmadas dessa musculatura, com relaxamento simultâneo do esfíncter externo, de maneira que o líquido da fossa prostática é impulsionado em sentido anterógrado pela uretra.

Em um artigo de revisão (29), a etiologia da ejaculação precoce primária foi postulada como estando associada à uma diminuição central do neurotransmissor serotonina e/ou à baixa sensibilidade do receptor 5-hidroxitriptamina (5-HT 2c) e/ou à hipersensibilidade de seu receptor (5-HT 1a).

Formulou-se a hipótese de limiar do tempo de ejaculação nesses pacientes, mediado pelo sistema serotoninérgico. A existência de um limiar baixo para a ejaculação, faria que esses homens suportassem durante pouco tempo a excitação sexual. Não importaria o que fizessem, sempre ejaculariam rapidamente, e isto estaria ligado à redução do neurotransmissor 5-HT (serotonina) e/ou à hipofunção do receptor de 5-HT 2c e/ou à hiperfunção do receptor de 5-HT 1a (30). Se o limiar de ejaculação fosse mais elevado, os homens teriam maior controle sobre o tempo até ejacular. Eles conseguiriam suportar prolongadamente a excitação sexual, e teriam a habilidade neurobiológica da decisão voluntária de ejacular em qualquer momento do ato sexual. Se o limiar fosse muito elevado, esses homens apresentariam dificuldades em ejacular e até mesmo não conseguiriam, mesmo se plenamente excitados sexualmente por uma ação inadequada da serotonina nos receptores 5-HT 2c e 5-HT 1a . Estes dados são antagônicos à idéia de que a ejaculação precoce seria 
um distúrbio adquirido por causa da ansiedade das primeiras relações sexuais, conforme sugerido por Masters e Johnson (6). Poderia-se crer que a ejaculação precoce seria uma variável de tempo mais curto para ejacular em alguns homens, com uma possível tendência familiar (30).

A conduta nos homens com ejaculação precoce inclui anamnese médica e sexual de cada paciente. Além da história médica, onde são obtidas informações sobre doenças agudas ou crônicas, cirurgias, uso de medicações e lesões genitais, realiza-se a anamnese sexual, quando se pergunta sobre o nível de entendimento sexual, o início da exposição às práticas sexuais, os mitos culturais ou religiosos e as práticas de masturbação. O exame físico detalhado abrange a pesquisa de sinais físicos de doenças crônicas, um breve exame neurológico, sinais de distúrbios endócrinos, características sexuais secundárias, exame dos testículos e presença de placas no pênis ou feridas (17).

Dentre as formas propostas de terapia comportamental, estão incluídas a manobra de Semans de pausar a penetração (31), a técnica de Masters e Johnson de pausa e compressão da glande (6) e o método de Kaplan de iniciar e interromper a penetração (32), que são tratamentos mecânicos e exigem grande cooperação da parceira. Muitos dos pacientes que respondem à essas técnicas, conseguindo uma melhora do tempo de latência da ejaculação, aparentemente não sustentam esses benefícios ao longo do tempo (33). 
Os tratamentos com cremes anestésicos tópicos na glande peniana foram uma modalidade medicamentosa inicialmente proposta, tendo sido utilizado creme de lidocaína com prilocaína 10 minutos antes da relação sexual (34).

O retardo da ejaculação é um efeito colateral observado em pacientes usando drogas antidepressivas desde o advento dos antidepressivos da classe dos triclíclicos e dos inibidores da monoamino oxidase (29). O uso de drogas orais veio preencher a lacuna dos pacientes desejosos de uma forma de terapia alternativa nos casos de insucesso com a psicoterapia, ou quando a parceira não era cooperativa. Essas drogas tem ação central e local, retardando o controle psiconeurológico da ejaculação, de um modo contínuo ou em momentos específicos ao seu uso.

O posterior surgimento dos antidepressivos inibidores da recaptação da serotonina, com menores efeitos colaterais e melhor tolerância do que os antidepressivos tricíclicos, ampliaram a possibilidade do uso dessa classe farmacológica. O aumento do tempo de latência foi observado com sertralina (20), paroxetina (35), fluoxetina (20) e clomipramina (36) que são os inibidores da recaptação da serotonina e ativam os receptores pós-sinápticos. Um desses receptores, o 5-HT 2c possui ações anorexígenas e ansiolíticas, além de aumentar o tempo para ejacular (16) (Figura 2). Essas drogas prolongam o tempo de latência da ejaculação de maneira sustentada, quando são usadas diariamente. A paroxetina em dose diária foi verificada como a droga que isoladamente promoveria a maior resposta em prolongar o tempo de ejaculação (35). 


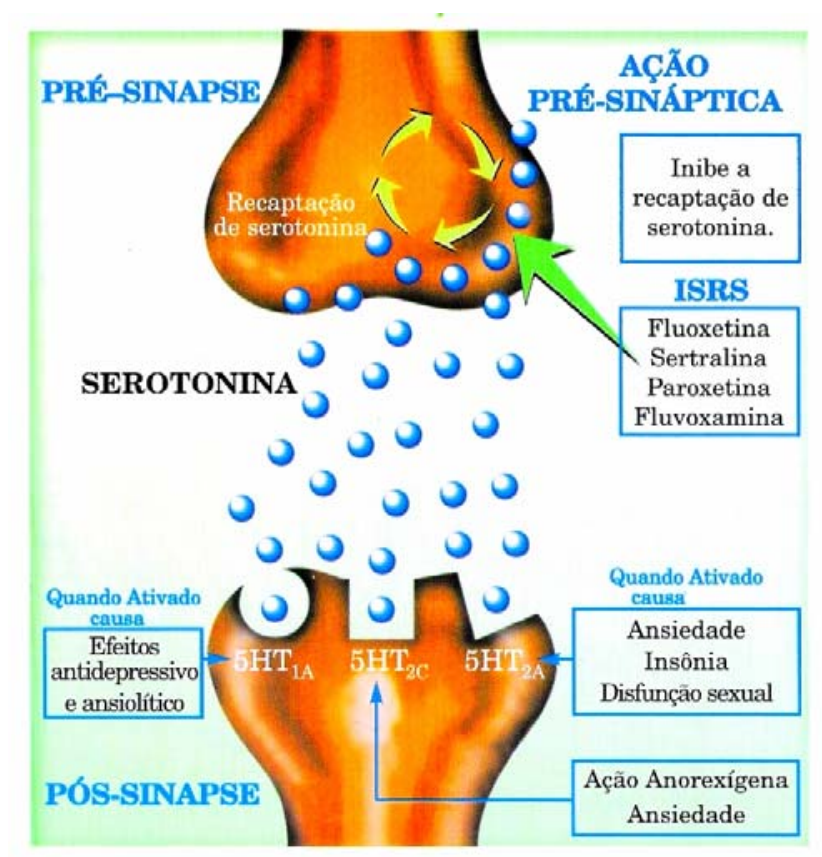

Figura 2: Ação dos antidepressivos inibidores de recaptação de seroronina na sinapse nervosa.

Novaretti et al. (37), estudaram pacientes em uso de fluoxetina $20 \mathrm{mg}$ em dose diária, e observaram melhora no tempo de ejaculação nos pacientes tratados, com poucos efeitos colaterais. Outra pesquisa demonstrou que a paroxetina é um tratamento eficiente e seguro em ejaculação precoce quando usada em doses diárias ou eventuais, próximas de um encontro sexual (38). A dificuldade na avaliação dos resultados obtidos nos tratamentos, é gerada pela ausência de métodos de aferição seguros nos trabalhos publicados. Um estudo relatou que a dose de $20 \mathrm{mg}$ de fluoxetina por dia seria a recomendada (39). Busch et al. Observaram experimentalmente que o uso de fluoxetina induziu a um aumento da contratilidade dos deferentes de ratos (40). A fluoxetina também foi testada com dose mais elevada, no tratamento da ejaculação precoce oferecendo bons resultados (41). 
Outra classe de medicamentos, esses mais recentes, dos inibidores da fosfodiesterase tipo 5, tem indicação aprovada para o tratamento da disfunção erétil, mas também promovem de um prolongamento do tempo de latência da ejaculação (42). Em estudos laboratoriais, foi observado que o uso dessas drogas teriam efeitos de ação periférica, que seriam: (a) modulação da resposta contrátil dos deferentes, das vesículas seminais, da próstata e da uretra, (b) indução de analgesia periférica e (c) prolongamento da duração total do tempo de ereção. Os mecanismos de ação central desses medicamentos seriam: (a) atuação central na via de ação do óxido nítrico / guanosina monofosfato (GMP) cíclico e (b) diminuição da ação central do sistema nervoso simpático (21).

O uso do inibidor de fosfodiesterase 5 sildenafila apresentou resultado no tratamento da ejaculação precoce em uso isolado (42) ou em combinação com inibidores de recaptação de serotonina, tanto com a paroxetina (23) ou com a sertralina (43).

A combinação de um antidepressivo inibidor da recaptação da serotonina junto com um inibidor da fosfodiesterase tipo 5 foi realizada associando a paroxetina e a sildenafila em um estudo (44) e a sertralina e a sildenafila em outro, com bons resultados (43). Um estudo utilizando somente a sildenafila, em uso eventual e próximo de uma relação sexual, mostrou que o tempo de latência da ejaculação aumentou mais do que com o uso isolado de paroxetina (15 minutos contra 4 minutos) (45). Neste estudo, a sildenafila isoladamente foi superior aos outros 
métodos de tratamento, em termos de tempo de latência ejaculatória e satisfação individual dos pacientes.

Alguns relatos da literatura tentam explicar o mecanismo em potencial responsável pelo prolongamento do tempo ejaculatório, quando se usa inibidores da fosfodiesterase tipo 5. Hull et al. Observaram em laboratório que o óxido nítrico poderia inibir a emissão da vesícula seminal em ratos, possivelmente por diminuição da atividade do sistema nervoso simpático (46). Por promover uma maior atividade do ciclo do GMPc, estimularia um efeito prolongado do óxido nítrico na emissão seminal. Kriegsfield et al. relataram que camundongos deficientes da enzima sintase endotelial do óxido nítrico (eNOS), apresentavam maior incidência de ejaculação precoce (47). Esta maior propensão para ejacular nesses animais, seria provavelmente relacionada à uma menor estimulação requerida para disparar a ejaculação. Camundongos completamente deficientes de eNOS, necessitavam de menos cópulas $(\mathrm{p}<0,003)$ e menos penetrações $(\mathrm{p}<0,001)$ para ejacular, quando comparados com camundongos sãos. Estes resultados sugerem que o óxido nítrico sintetizado pela eNOS pode estar envolvido na fisiologia da ejaculação. Heuer et al. observaram in vitro, que a contração da vesícula seminal humana é regulada parcialmente pela cascata GMPc-óxido nítrico (48). Esta constatação justificaria o uso de inibidores da fosfodiesterase, nos distúrbios ejaculatórios associados com hiperexcitação. 


\section{JUSTIFICATIVA E OBJETIVOS:}

O uso de drogas orais da classe dos inibidores da fosfodiesterase tipo 5 e os antidepressivos, usados em isolado ou em associação, tem sido empregados no tratamento da ejaculação precoce. No entanto, nenhuma droga foi aprovada por agências reguladoras especificamente para esse fim, até o presente.

O objetivo deste estudo foi avaliar se o uso oral de tadalafila, que é um inibidor da fosfodiesterase-5, associado a fluoxetina $90 \mathrm{mg}$, que é um anti-depressivo da classe dos inibidores da recaptação seletiva de serotonina, em apresentação de liberação gradual com uma dose semanal, poderia prolongar o tempo até atingir o orgasmo em homens com queixa de ejaculação precoce primária. 


\section{PACIENTES E MÉTODOS:}

\section{Casuística:}

Sessenta pacientes com ejaculação precoce primária foram admitidos no estudo em regime de atendimento ambulatorial no Posto de Atendimento Médico Hélio Pellegrino da Secretaria Municipal de Saúde do Rio de Janeiro. O estudo foi aprovado pelo Comitê de Ética para Análise de Projetos de Pesquisa (CAPPesq) da Universidade de São Paulo, sob o protocolo número 998/2003, e seguiu as diretrizes da Resolução número 196/1996 sobre pesquisa envolvendo seres humanos do Conselho Nacional de Saúde. Os pacientes leram e assinaram o termo de consentimento informado, aceitando em participar no estudo e foram advertidos dos possíveis efeitos colaterais das drogas.

As reações adversas esperadas e notificadas no consentimento informado eram: cefaléia, dispepsia, lombalgia, mialgia, congestão nasal, rubor facial, tontura, edema de pálpebra, hiperemia conjuntival, secura da boca, sudorese, calafrios, erupções de pele, diarréia, náusea, vômito, tremor, anorexia, ansiedade, sonolência, distúrbios do sono, bocejo, visão anormal, priapismo e anormalidades da miccção. 
Todos pacientes eram heterossexuais entre 24 e 64 anos de idade, com relacionamento estável com a mesma parceira nos últimos seis meses, no mínimo. Os critérios de exclusão foram: pacientes em uso de nitratos, issossorbida, nitroglicerina, com insuficiência hepática ou renal e pacientes com doença cardíaca para os quais a atividade sexual não fosse aconselhada, uso de outras drogas ativas do sistema nervoso central, como fenitoína, carbamazepina, haloperidol, clozapina, diazepam, alprazolam, lítio e imipramina, uso de varfarina, cetoconazol, uso de inibidores da monoamina-oxidase, diabéticos, indivíduos com hipersensibilidade aos princípios ativos das duas medicações, uso abusivo de bebidas alcoólicas, e uso de opióides ou drogas ilícitas.

O estudo clínico foi prospectivo, duplo-cego e randomizado. Na primeira visita os pacientes eram entrevistados e avaliados em relação a possibilidade e aceitação em participar no estudo. Os pacientes foram avaliados de forma preliminar com anamnese, exame físico, história sexual e preenchimento do questionário Internacional do Índice de Função Erétil (IIEF) (49), respondendo à três perguntas escolhidas das quinze que compõem o questionário. Este é um questionário mundialmente utilizado em estudos de disfunção erétil e sexual.

Desse questionário, as 3 perguntas escolhidas para avaliação abordavam especificamente a satisfação da vida sexual, e são as seguintes:

Pergunta 7: “Quando houve relação sexual, com que frequência foi satisfatória para você ?”,

Pergunta 13: “Qual sua satisfação em relação à sua vida sexual ?” 
Pergunta 14: “Qual sua satisfação sexual ultimamente com sua parceira?”

As respostas possíveis eram: 1 = quase nunca; 2 = poucas vezes; 3 = algumas vezes; 4 = maioria das vezes; 5 = quase sempre.

Foram orientados a aferir o tempo de ejaculação, uma vez por semana durante três semanas, utilizando um cronômetro digital de pulso, da mesma marca e modelo, que foi entregue a cada um dos pacientes junto com um diário para anotação destes tempos. Esses valores iniciais serviriam como o tempo basal para a comparação com o tempo depois de estarem em uso dos medicamentos. O tempo de latência para ejaculação em penetração vaginal, do termo inglês intravaginal ejaculatory latency time é definido pelo tempo do começo da penetração vaginal até o início da emissão da ejaculação.

Os participantes foram distribuídos aleatoriamente em 4 grupos de 15 pessoas cada e tanto o pesquisador como os pacientes desconheceram qual grupo foi designado para medicamentos ou placebo.

Os 4 grupos fizeram uso das cápsulas na seguinte divisão:

Grupo 1: placebo associado a fluoxetina $90 \mathrm{mg}$

Grupo 2: tadalafila $20 \mathrm{mg}$ associado a fluoxetina $90 \mathrm{mg}$

Grupo 3: tadalafila $20 \mathrm{mg}$ associado a placebo

Grupo 4: placebo associado a placebo (controle) 
As cápsulas com cada princípio ativo e o seu placebo eram rigorosamente iguais, e foram preparadas pela Farmácia Galênica (Rua Barata Ribeiro, 406-A, Rio de Janeiro - RJ, CEP 22040-000ＣNPJ 29.778.339/0001-67 - farmacêutica responsável Adriana V. Venture CRF 5.221). As cápsulas de tadalafila (e seu placebo), e de fluoxetina (e seu placebo) eram diferentes entre si. Os pacientes receberam dois envelopes, cada envelope com uma cápsula de fluoxetina (ou placebo) e em outro envelope tadalafila (ou placebo). Eram orientados a usar a cápsula de fluoxetina (ou placebo) uma vez por semana, sempre no mesmo dia da semana e mesmo horário da primeira dose. A cápsula de tadalafila (ou placebo) era para ser usada em período entre 1 hora e 36 horas precedendo a atividade sexual, que é o período de atuação da medicação.

Os pacientes anotaram em diário os tempos da ejaculação destas relações sexuais, utilizando o mesmo cronômetro das anotações iniciais. Os efeitos adversos também foram anotados no mesmo diário.

Orientou-se aos pacientes que tentassem manter as relações sexuais o mais próximo possível das características habituais, não tentando interromper o desejo de ejacular ou aumentar e diminuir a velocidade da intromissão vaginal. Caso usassem preservativos habitualmente, também deveriam usá-los nas relações sexuais durante o estudo. Nos casos de mais de uma relação em um mesmo encontro, somente o tempo da primeira ejaculação era aferido e anotado. Nenhum acompanhamento psicológico foi utilizado neste estudo. 
Os pacientes foram avaliados em intervalos de três semanas após o início do protocolo, totalizando 5 visitas.

Repetiu-se o fornecimento das cápsulas na $6^{\mathrm{a}}$ e $9^{\mathrm{a}}$ semanas desde o início do estudo, e avaliou-se os efeitos, além de não espaçar muito o acompanhamento. Nestas visitas os pacientes retornaram as anotações do diário, de tempo e efeitos colaterais.

Na última visita, as mesmas três perguntas do questionário Internacional do Índice de Função Erétil eram oferecidas para respostas e anotadas, para posterior comparação com as inicialmente respondidas. 


\section{Avaliação Estatística:}

Os padrões de eficácia foram comparados entre grupos com análise de variância (ANOVA) (50). As médias dos tempos antes do tratamento foram chamadas de Tempo Inicial (TI) e as médias dos tempos com o tratamento foram chamadas de Tempo Final (TF). Para uniformidade dos cálculos, as anotações de tempo foram também analisadas em graduação de logaritmo (LOG), fornecendo maior linearidade à esta variável. O gradiente da diferença entre a média do Tempo Final e média do Tempo Inicial foi chamada de Delta (variação), e comparada entre os grupos também utilizando-se ANOVA. O teste de Tukey foi utilizado para comparar as médias entre os 4 grupos. O teste não-paramétrico H de Kruskal-Wallis (análogo da ANOVA) foi utilizado para avaliar o p valor na suposição de que a amostra não tivesse uma distribuição normal. As variáveis quantitativas foram resumidas com média e desvio-padrão, e as variáveis qualitativas resumidas com números e percentuais. Para todas as avaliações estatísticas foi considerado como significativamente diferente os resultados menores que $0,05(\mathrm{p}<0,05)$. O pacote estatístico utilizado para confecção das análises e gráficos foi o SPSS (Statistical Package for Social Sciences) para Windows versão 12.0. 


\section{RESULTADOS:}

Entre a média das idades dos doentes dos 4 grupos e na média do tempo inicial de ejaculação, não houve diferença estatisticamente significativa (tabela 1).

A idade média dos homens foi 45,4 anos (desvio-padrão +/- 9,6) com variação entre 24 e 64 anos. A média do tempo basal de latência ejaculatória, ou seja, antes do tratamento foi 51,3 segundos (desvio-padrão +/- 23,2 segundos), com variação entre 5,33 segundos e 85,67 segundos. As médias dos tempos basais não diferiu estatisticamente entre os grupos $(\mathrm{p}=0,805)$ (tabela 1$)$.

O gradiente de diferença (variação) entre a média do logaritmo do tempo inicial e a média do logaritmo do tempo final foi avaliado, e chamado Delta. O resultado ANOVA unidirecional do Delta entre os grupos foi estatisticamente significativo $(\mathrm{p}<0,001)$ (tabela 2 e figura 3$)$.

Tabela 1 - Idade e tempo de ejaculação inicial dos pacientes nos 4 grupos

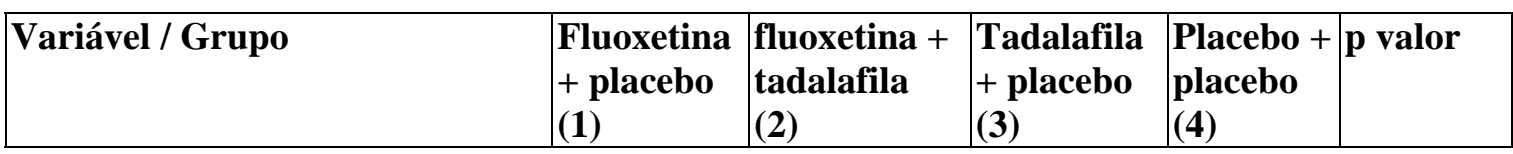

\begin{tabular}{|l|c|c|c|c|c|}
\hline idade (anos) & 50 & 42,81 & 43,2 & 45,93 & 0,152 \\
\hline Média do tempo inicial (segundos) & 56,55 & 49,57 & 49,26 & 49,86 & 0,805 \\
\hline
\end{tabular}

Tabela 2 - Tempo após tratamento e Delta (variação) entre os tempos nos 4 grupos

\begin{tabular}{|l|l|l|l|l|l|}
\hline Variável / Grupo & $\begin{array}{l}\text { Fluoxetina } \\
+ \text { placebo } \\
(\mathbf{1})\end{array}$ & $\begin{array}{l}\text { Fluoxetina }+ \\
\text { tadalafila } \\
(2)\end{array}$ & $\begin{array}{l}\text { Tadalafila } \\
+ \text { placebo } \\
(3)\end{array}$ & $\begin{array}{l}\text { Placebo + } \\
\text { placebo } \\
(4)\end{array}$ & p valor \\
\hline
\end{tabular}

\begin{tabular}{|l|l|l|l|l|l|}
\hline Média do tempo final (segundos) & 230,98 & 464,41 & 207,11 & 64,33 & $0,003^{*}$ \\
\hline Delta (variação em segundos) & 174,42 & 414,83 & 160,05 & 18,71 & $0,003^{*}$ \\
\hline
\end{tabular}

* estatisticamente significativo 


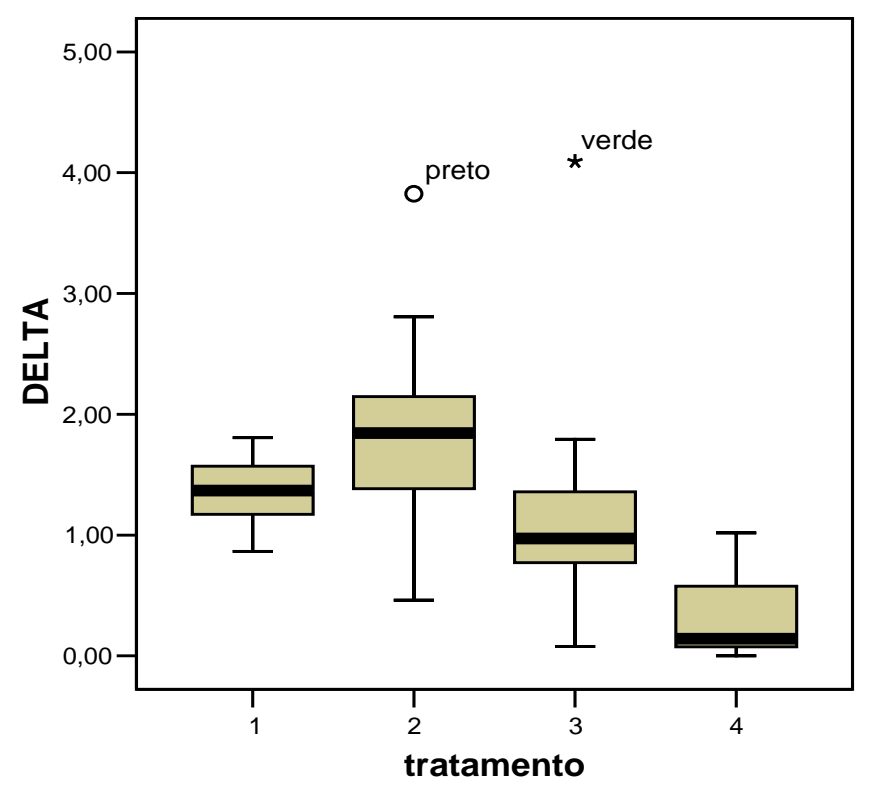

Figura 3: Diferença do tempo final e tempo inicial em representação logaritmica. 1) fluoxetina + placebo; 2) fluoxetina + tadalafila; 3) tadalafila + placebo; 4) placebo + placebo $(\mathbf{p}<\mathbf{0 , 0 0 1})$

A média do tempo final de latência ejaculatória, aquele que foi obtido ao final do tratamento, foi comparado entre os grupos usando análise de variância (ANOVA unidirecional), com resultado estatisticamente significativo demonstrando diferença entre os grupos $(\mathrm{p}<0,001)$ (Tabela 2$)$.

Foi realizado para o tempo final, o teste estatístico H não-paramétrico de Kruskal-Wallis, que é o teste análogo do ANOVA para análise de amostras independentes, em caso de uma suposta não normalidade da amostra, e o resultado também foi estatisticamente significativo $(\mathrm{p}<0,001)$.

Efetuamos o teste de Tukey, que é utilizado para avaliar as diferenças pareadas antes e depois do tratamento entre cada grupo separadamente Os gradientes dos tratamentos (variações entre os tempos) comparados com o grupo placebo 
apresentaram os seguintes resultados: grupo (1) fluoxetina ( $\mathrm{p}=0,001)$, grupo (2) tadalafila + fluoxetina $(\mathrm{p}<0,001)$ e grupo (3) tadalafila $(\mathrm{p}=0,008)$. Todos foram estatisticamente superiores ao resultado com placebo, com maior significância para o tratamento combinado com as duas drogas.

O aumento do tempo final em relação ao tempo inicial (incremento de tempo) foi da ordem de 3,08 vezes para o grupo que usou a fluoxetina (grupo 1), de 8,36 vezes para o grupo que usou a associação de tadalafila e fluoxetina (grupo 2) e de 3,2 vezes para o grupo que usou a tadalafila (grupo 3). O placebo apresentou aumento de 0,29 vezes (tabela 3 e figuras 4 e 5 )

Tabela 3 - Aumento relativo do tempo de ejaculação nos pacientes após tratamento com diferentes medicamentos.

\begin{tabular}{|l|l|l|ll|}
\hline Grupos & tempo inicial & tempo final & aumento relativo & $\mathbf{p}$ \\
\hline
\end{tabular}

\begin{tabular}{|l|l|l|lr|}
\hline Fluoxetina + placebo (1) & $56,55 \mathrm{~s}$ & $230,98 \mathrm{~s}$ & fator 3,08 x & $<0,001$ \\
\hline fluoxetina + tadalafila (2) & $49,57 \mathrm{~s}$ & $464,41 \mathrm{~s}$ & fator 8,36 x & $<0,001$ \\
\hline Tadalafila + placebo (3) & $49,26 \mathrm{~s}$ & $207,11 \mathrm{~s}$ & fator 3,20 x & 0,001 \\
\hline Placebo + placebo (4) & $49,86 \mathrm{~s}$ & $64,33 \mathrm{~s}$ & fator 0,29 x & 0,042 \\
\hline
\end{tabular}




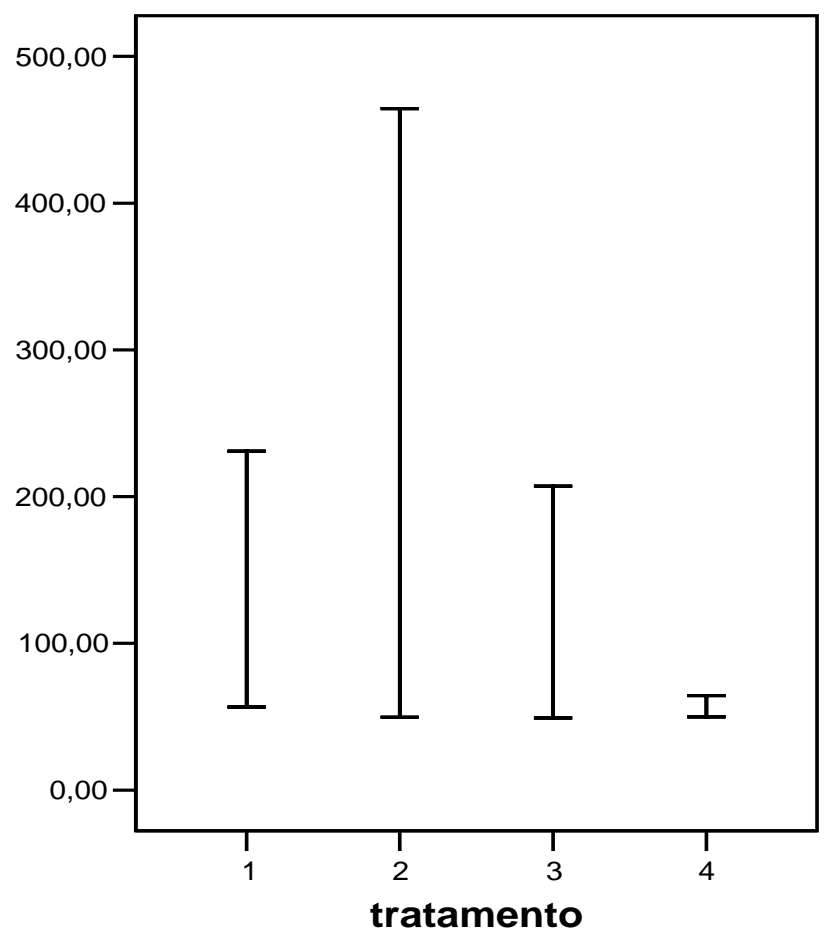

Figura 4: Gradiente de diferença entre o tempo final e o tempo inicial (em segundos)

1) fluoxetina + placebo; 2) tadalafila + fluoxetina; 3) tadalafila + placebo; 4) placebo + placebo

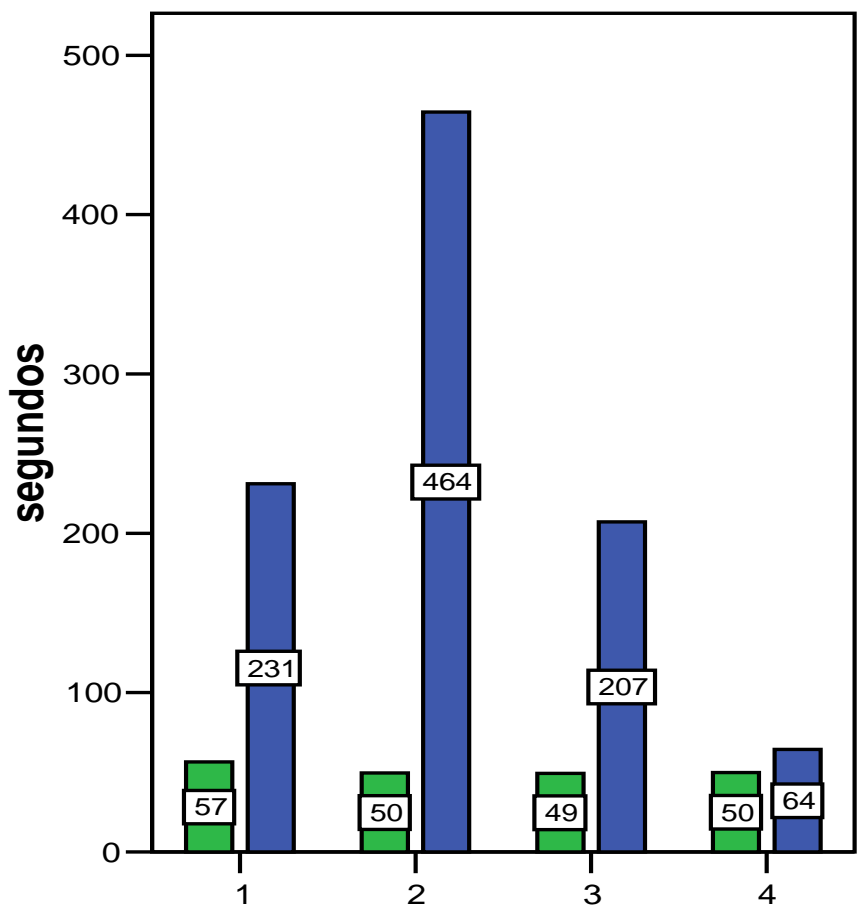

Figura 5: Média do tempo inicial em segundos (coluna verde) e média do tempo final (coluna azul)

1) fluoxetina + placebo; 2) tadalafila + fluoxetina; 3) tadalafila + placebo; 4) placebo + placebo 
Quando efetuado o teste $t$ de Student intragrupos, observou-se o p valor da correlação do logaritmo da média do tempo inicial e o logaritmo da média do tempo final. Os resultados foram: fluoxetina (grupo 1) $\mathrm{p}<0,001$, fluoxetina + tadalafila (grupo 2) $\mathrm{p}<0,001$, tadalafila (grupo 3) $\mathrm{p}=0,001$ e placebo (grupo 4) $\mathrm{p}=0,042$.

Inclusive o grupo placebo mostrou discreta melhora, apesar de próximo do nível de corte para significância, o que mostra que ocorreu efeito placebo nesse grupo que não usou nenhum princípio ativo, porém muito pequeno quando comparado com os outros grupos.

Os resultados do questionário IIEF foram avaliados para as perguntas de avaliação subjetiva: respondidos antes e após o tratamento. Para o teste IIEF, as médias dos aumentos na pontuação final foram: (grupo 1) fluoxetina (3,46 pontos), (grupo 2) tadalafila + fluoxetina (5,2 pontos), (grupo 3) tadalafila (2,8 pontos) e (grupo 4) placebo (0,4 pontos). Também na avaliação subjetiva dos resultados houve maior aumento no grupo que usou as drogas em associação (figura 6).

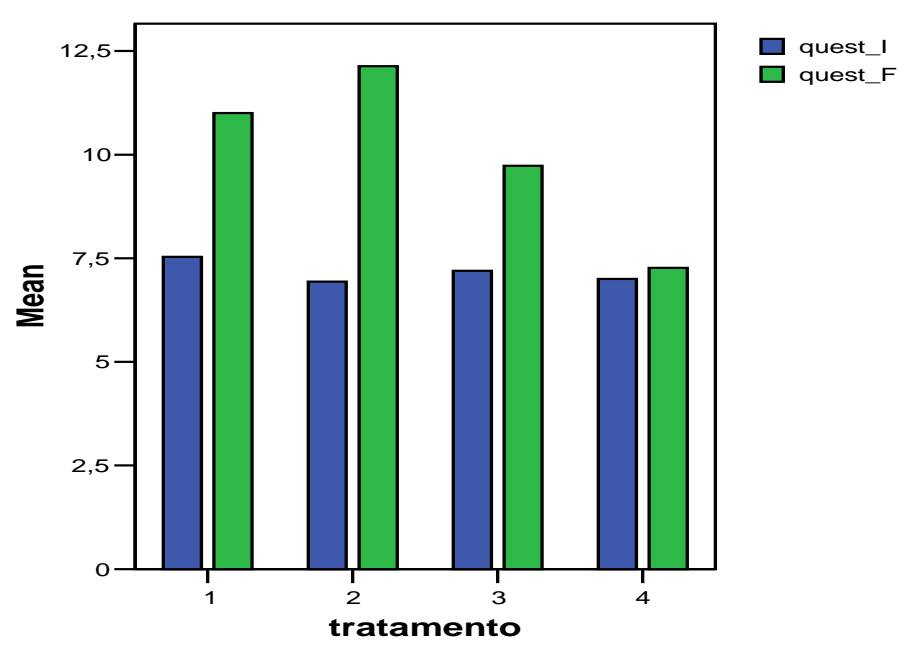

Figura 6: Respostas do Questionário do Índice Internacional da Função Sexual: antes do tratamento (coluna azul) e depois do tratamento (coluna verde).

1) fluoxetina + placebo; 2) tadalafila + fluoxetina; 3) tadalafila + placebo; 4) placebo + placebo 
Reações adversas foram observadas nos grupos usando medicamentos e placebo:

Grupo 1 (fluoxetina): em cinco pacientes (33\%). Sonolência e bocejos (3/5), náusea (1/5), astenia (3/5);

Grupo 2 (fluoxetina + tadalafila): em seis pacientes (40\%). Sonolência e bocejos (3/6), palpitação(1/6), dor muscular(1/6), náusea (2/6)

Grupo 3 (tadalafila): em quatro pacientes (26\%). Cefaléia (3/4), rubor facial (2/4), palpitação (2/4);

Grupo 4 (placebo) em dois pacientes (12\%). Diarréia (1/2) epigastralgia $(1 / 2)$.

Um paciente que pertencia ao grupo que estava usando fluoxetina mais placebo saiu do estudo. Sentiu forte náusea e ansiedade após ingerir a primeira cápsula de fluoxetina, tendo comunicado ao pesquisador imediatamente o efeito colateral e a opção por não prosseguir no estudo. 


\section{DISCUSSÃO:}

Poucos estudos foram publicados sobre tratamentos da ejaculação precoce com uso de antidepressivos em uso eventual, próximos à um encontro sexual $(20,51,52,53,54)$. Esses antidepressivos usados um pouco antes da relação, podem promover um efeito favorável, mas precisariam continuar sendo usados com frequência diária, para manutenção de sua concentração sérica.

Dentre os antidepressivos inibidores da recaptação da serotonina usados no tratamento da ejaculação precoce, a paroxetina é a que provoca o maior aumento no tempo da latência ejaculatória (43). No entanto, a paroxetina, a sertralina e a fluoxetina podem promover efeitos indesejados como fadiga, bocejos frequentes, náusea moderada, diarréia e transpiração excessiva, no entanto, esses efeitos tendem a desaparecer após 3 semanas, enquanto os efeitos de retardo da ejaculação se manifestam a partir da segunda semana, ou mesmo antes (39).

Uma meta-análise sobre ejaculação precoce (33) avaliou 79 estudos levantados em um período de 60 anos (1943 - 2003). Estes estudos incluíam diferentes metodologias de avaliação dos pacientes, além de uso de drogas diversas. Dentre todos os estudos, apenas oito trabalhos (18,5\%) preenchiam os critérios considerados adequados para obtenção de conclusões. Eram estudos prospectivos, randomizados e duplo-cegos com grupo controle. 
Foi demonstrado que pacientes tratados somente com a sildenafila conseguiram prolongar o tempo de latência ejaculatória (42), mas não existem estudos realizados usando a tadalafila, que foi utilizada em nosso estudo, ou a vardenafila, que são os outros inibidores da fosfodiesterase tipo 5.

A tadalafila produz resposta terapêutica em pacientes com disfunção erétil psicogênica, e esperaria-se que algum efeito benéfico também pudesse ser obtido em pacientes com ejaculação precoce com componente psicogênico. Balon (55) relatou que o tempo de latência ejaculatória é dependente de uma ereção plena, e a melhora da ereção teria um efeito positivo em pacientes com ejaculação precoce.

O efeito central da tadalafila, através do óxido nítrico no reflexo da ejaculação, seria outro mecanismo de ação na melhora da ejaculação precoce. Enzimas de fosfodiesterase foram encontradas em cérebros de ratos, e o óxido nítrico, que é o mensageiro intracelular da tadalafila, é atuante em inúmeras funções fisiológicas, sendo uma delas a comunicação neural do cérebro (56). A atividade do óxido nítrico na área pré-óptica medial inibiria a ejaculação por redução do tônus simpático (57).

Uma nova droga, a dapoxetina, vem sendo estudada especificamente para o tratamento da ejaculação precoce. Esta droga é um inibidor da recaptação da serotonina de rápido início de ação e meia-vida curta. Um estudo em fase II testou a droga nas doses de $60 \mathrm{mg}$ e $100 \mathrm{mg}$ (58) e comparadas com placebo em 130 pacientes. O aumento do tempo inicial foi superior no grupo que fez uso na dose de 
100 mg, mas também foi o que apresentou mais efeitos colaterais. A conclusão foi que a dose de 60 mg seria eficiente e tolerável, melhorando o sintoma à partir da primeira dose.

A associação das duas drogas, o antidepressivo inibidor da recaptação de serotonina e o inibidor da fosfodiesterase tipo 5, poderia apresentar resultados favoráveis por algumas suposições. A enzima CYP2D6 é muito importante no metabolismo microssomal dos inibidores de recaptação da serotonina, e esta enzima contribui significativamente na absorção de inibidores de fosfodiesterase tipo 5, como a tadalafila, potencializando seu efeito. A relação das drogas com a inibição do citocromo P450 poderia ser um fator importante na interação das duas drogas, quando usadas simultaneamente (21).

O uso do cronômetro para a avaliação dos tempos pelos pacientes em nosso estudo, foi uma forma de obter com maior precisão a variável de tempo, de maneira que os pacientes não se baseassem somente em avaliações subjetivas. A avaliação subjetiva foi avaliada somente pelas respostas do questionário do Índice Internacional de Função Erétil, assinaladas antes e após o tratamento.

O método da avaliação do tempo da ejaculação feito com cronômetro a cada relação, é um melhor parâmetro para efeito de comparações . A utilização dessa metodologia permite a elaboração de artigos considerados como medicina baseada em evidências. Não são aconselhados os estudos abertos, retrospectivos, e com 
avaliações subjetivas da melhora do tempo da ejaculação. Estudos retrospectivos com avaliações subjetivas tem menor valor científico que os prospectivos (17)

Nosso estudo avaliou o uso combinado de tadalafila com a fluoxetina de liberação lenta, com atenção particular para alguns pontos específicos. Foi notado um efeito benéfico em relação ao prolongamento do tempo para ejaculação, nos grupos que usaram a fluoxetina isolada ou com a tadalafila, com maior tempo para ejacular em relação ao tempo inicial de base no grupo que associou as duas medicações $(\mathrm{p}<0,001)$.

O presente estudo demonstrou também que a associação de tadalafila com fluoxetina de liberação lenta foi capaz de produzir um efeito consistente no retardamento da ejaculação em homens com ejaculação precoce. O uso puro de fluoxetina apresentou um efeito superior ao placebo, mas quando avaliamos a média dos tempos iniciais, antes do tratamento, comparado com a média dos tempos em uso de medicamentos, o maior gradiente de diferença foi no grupo que usou a combinação das drogas.

Um aspecto passível de discussão de nosso trabalho é em relação ao uso de fluoxetina na dose de $90 \mathrm{mg}$ sem a titulação da dose, que deveria ser aumentada gradativamente, conforme é proposta em psiquiatria no tratamento da depressão (59). A apresentação de 90 mg de liberação semanal de fluoxetina, foi demonstrada como eficaz e tolerada em estudos anteriores (60). Os microgrânulos dessa apresentação da droga possuem um revestimento que retarda a liberação entérica no trato digestivo, 
em região onde o pH é maior que 5,5. Deste modo, a absorção da droga torna-se gradual e equivaleria ao uso na dose diária de 20 mg em relação à concentração sérica (60). A tolerância da apresentação de liberação lenta foi a mesma da apresentação diária, assim como os efeitos colaterais, sendo um pouco menores na apresentação de liberação lenta (61). Um efeito observado em um estudo nos pacientes usando esta nova apresentação, foi o relato de melhora da função sexual em 40,6\% dos homens (62).

Em nosso estudo não foi feita a titulação da dosagem da fluoxetina, que foi iniciada na dose de tratamento com 90 mg, e isto não demonstrou aumento dos efeitos colaterais. Os efeitos foram comparáveis com outro estudo em que se utilizou a mesma dosagem para fins de tratamento da ejaculação precoce (41). 


\section{CONCLUSÃO:}

O aumento do tempo de ejaculação em pacientes com queixa de ejaculação precoce foi fortemente influenciado quando se usou fluoxetina 90 mg de liberação lenta uma vez por semana associada a tadalafila 20 mg antes da relação sexual (8,36 vezes) quando comparada ao uso de placebo antes das relações sexuais (0,29 vezes). 


\section{REFERENCIAS:}

1 McMahon, CG; Abdo, C; Incrocci, L; Perelman, M; Rowland, D; Waldinger, M e Xin, ZC. Disorders of orgasm and ejaculation in men. J Sex Med 2004; 1: 58-65

2 St. Lawrence, JS; Madakasira, S. Evaluation and treatment of premature ejaculation: A critical review. Int J Psychiatry Med. 1992; 22 :77-97

3 Cooper, AJ. A Factual study of male potency dysorders. Br J Psychiatry 1968, 114: $719-731$

4 Marmor, J. Impotence and Ejaculatory Disturbances. In: Sadock, JB, Kaplan, HI, Freedman, AM, eds. The Sexual Experience. Baltimore, MD: Williamm \& Wilkins, 1966

5 Colpi, GM, Gsnviullsvvi, G, Beretta, G, Negri, L, Zanollo, A. Evoked sacral potentials in subjects with true premature ejaculation. Andrologia. 1986; 18: $583-6$

6 Masters, WH e Johnson, VE: Human Sexual Inadequacy. Boston: Little, Brown and Co., 1970 
7 Kaplan, HS: The New Sex Therapy: Active Treatment of Sexual Dysfunctions. New York: Brunner/Maazel, 1974

8 Diagnostic and Statistical Manual of Mental Disorders, DSM-IV, 4th ed., text revision. Washington, D. C.: American Psychiatric Association, 2000

9 Godpodinoff, ML: Premature ejaculation: clinical subgroups and etiology. $J$ Sex Marital Ther 1989, 15: 130-134

10 Laumann, EO, Paik, A, Rosen, RC: Sexual dysfunction in the United States: prevalence and predictors. JAMA 1999. 281: 537-44

11 Simons, JS, Carey, MP: Prevalence of sexual dysfunctions: results from a decade of research. Arch Sex Behav 2001, 30: 177-219

12 Aschka, C, Himmel, W, Ittner, E, Kochen, MM: Sexual problems of male patients in family practice. J Fam Pract 2001, 50: 773-8

13 Frank, E, Anderson, C, Rubinstein, D: Frequency of sexual dysfunction in normal couples. $N$ Engl J Med 1978, 299: 111- 114

14 Metz, ME, Pryor, JL, Nesvacil, LJ, Abuzzahab, F, Sr, Koznar, J: Premature ejaculation: a psychophysiological review. J Sex Marital Ther 1997, 23: 3-23 
15 Abdo, CHN. Estudo da Vida Sexual do Brasileiro. São Paulo, SP. Editora Bregantini, $1^{\mathrm{a}}$ edição, 2004

16 Waldinger, MD. Lifelong premature ejaculation: from authority-based to evidence-based medicine. BJU Int 2004, 93: 201-7

17 Montague, DK, Jarow, JJ, Broderick, GA, Dmochowski, RR, Heaton, PW, Lue, TF et al. AUA Guideline on the pharmacologic management of premature ejaculation. J Urol 2004. 172: 290-4

18 Kilmann, PR, Auerbach, R. Treatment of premature ejaculation and psychogenic impotence: a critical review of the literature. Arch Sex Behav 1979, 8:81-100

19 Xin, ZC, Choi, YD, Lee, SH, Choi, HK. Efficacy of a topical agent SS-cream in the treatment of premature ejaculation: preliminary clinical studies. Yonsei Med J 1997; 38: 91-5

20 Kim, SC, Seo, KK. Efficacy and safety of fluoxetine, sertraline and clomipramine in patients with premature ejaculation: a double-blind, placebo controlled study. J Urol 1998, 159: 425-7 
21 Abdel-Hamid, IA. Phosphodiesterase 5 Inhibitors in Rapid Ejaculation Potential use and possible mechanisms of action. Drugs, 2004. 64 : 13-26

22 Chen, J, Mabjeesh, NJ, Matzkin, H, Greenstein, A. Efficacy of sildenafil as adjuvant therapy to selective serotonin reuptake inhibitor in alleviating premature ejaculation. Urology 2003 61: 197-200

23 Salonia, A, Maga, T, Colombo, R, Scattoni, V, Briganti, A, Cestari, A, et al. A prospective study comparing proxetine alone versus paroxetine plus sildenafil in patients with premature ejaculation. $J$ Urol 2002, 168: 24869

24 Gross, S. Practical treatise on impotence and sterility. Edinburgh: Y.J. Pentland, 1887 (apud Waldinger 2004, ref. 16)

25 Kraft-Ebing, RF. Psychopathia Sexualis. $11^{\mathrm{a}}$ Ed. Stuttgart: Publishing Hause Enke, 1901 (apud Waldinger 2004, ref. 16)

26 Abraham, K. Ejaculatio praecox. Z. Fur. Aerztliche Psychoanalyse (4): 171, 1917 (apud Waldinger 2004, ref. 16)

27 Schapiro, B. Premature ejaculation. A Review of 1130 Cases. J Urol 1943, 50: 374-9 (apud Waldinger 2004, ref. 16) 
28 Witt, MA, Grantmyre, JE. Ejaculatory failure. World J Urol 1993, 11:89-95

29 Waldinger, MD, Berendsen, HHG, Blok, BFM, Olivier, B, Holstege, G Premature ejaculation and SSRI-induced delayed ejaculation: the involvement of the serotoninergic system. Behav Brain Res 1998, 92:111-8

Waldinger, MD. The neurobiological approach to premature ejaculation. J Urol 2002, 168: 2359-67

31 Semans, JR Premature ejaculation: a new approach. South Med J 1956, 49:3538

32 Seftel, AD, Althof, SE. Premature ejaculation. Em Mulcahy, J.J. ed. Diagnosis and Management of Male Sexual Dysfunction. New York, NY: IgakuShoin; 196-203, 1997

Waldinger, MD, Zwinderman, AH, Schweitzer, DH, Olivier, B. Relevance of methodological designs for the interpretation of efficay of drug treatment of premature ejaculation: a systematic review and meta-analysis. Int $J$ Impot Res 2004, 16:369-81

34 Berkovitch, M, Keresteci, AG, Koren, G. Efficacy of prilocaine-lidocaine cream in the treatment of premature ejaculation. J Urol 1995, 154:1360-1 
Waldinger MD, Hengeveld MW, Zwinderman AH. Ejaculation-retarding properties of paroxetine in patients with primary premature ejaculation: a double-blind, randomized, dose-response study. Br J Urol 1997, 79:5925

36 Seo, KK, Kim, SC, Lee, MY. Comparison of peripheral inihibitory effects of clomipramine with selective serotonin re-uptake inhibitors on contraction of vas deferens: in vitro and in vivo studies. $J$ Urol 2001, 165: 2110-4

37 Novaretti, JPT, Pompeo, ACL, Arap, S. Selective serotonin uptake inhibitor in the treatment of premature ejaculation. Braz J Urol 2002, 28: 116-22

McMahon, CG, Touma, K. Treatment of premature ejaculation with paroxetine hydrochloride as needed: 2 single-blind placebo controlled crossover studies. J Urol 1999, 161: 1826-1830

39 Kaplan, PM: The use of serotoninergic uptake inhibitors in the treatment of premature ejaculation. J Sex Marital Ther 1994, 20: 321-4

40 Busch, L, Wald, M, Borda, E. Long-term treatment with fluoxetine associates with less peripheral effects on rat vas deferens contractility. Life Sci 1999; 64: 117 
41 Manasia, P, Pomerol, J, Ribé, N, Pozo, RG, Garcia, JA. Comparison of the efficacy and safety of $90 \mathrm{mg}$ versus $20 \mathrm{mg}$ fluoxetine in the treatment of premature ejaculation. J Urol 2003, 170: 164-5

42 Lobik, L, Cytron, S, Kravich, S, Paz, A. The effect of sildenafil citrate in the treatment of secondary rapid ejaculation. J Urol 2003, 169 (Suppl.): 378

43 Lozano, AF, Castane, ER. Premature ejaculation: sildenafil plus sertraline versus sertraline alone and sildenafil alone. J Urol 2003, 169 (Suppl.): 247

44 Hirsch, M, Donatucci, C, Glina, S, Montague, D, Montorsi, F, Willie, M. Standards for clinical trials in male sexual dysfunction: Erectile dysfunction and rapid ejaculation. J Sex Med 2004, 1: 87-91

45 Abdel-Hamid, IA, El Naggar, EA, El Gilany, AH. Assessment of as needed use of pharmacotherapy and the pause-squeeze technique in premature ejaculation. Int J Impot Res 2001, 13: 41-5

Hull, EM, Lumley, LA, Matuszewich, L, Dominguez, J, Moses, J, Lorrain, DS. The roles of nitric oxide in sexual function of male rats. Neuropharmacology 1994, 33:1499-504 
47 Kriegsfeld, LJ, Demas, GE, Huang, PL, Burnett, AL, Nelson, RJ. Ejaculatory abnormalities in mice lacking the gene for endothelial nitric oxide synthase (eNOS -/-). Physiol Behav 1999, 67:561-6

Heuer, O, Uckert, S, Machtens, SA, Stief, CG, Tsikas, D, Frolich, JC et al. Effects of various nitric oxide donating agents on the contractility and cyclic nucleotide turnover of human seminal vesicles in vitro. Urology 2002, 59: 958-62

Rosen, RC, Riley, A, Wagner, G, Osterloh, IH, Kirkpatrick, J, Mishra, A. The international index of erectile dysfunction (IIEF): a multidimensional scale for assessment of erectile dysfunction. Urology 1997, 49: 822-30

Bisquerra, R, Sarriera, JC e Martinez, F. Introdução à estatística. Enfoque informático com o pacote estatístico SPSS, 1ª Ed. Artmed Ed. 115-137, 2004

51 Segraves, RT, Saran, A, Segraves, K, Maguire, E. Clomipramine versus placebo in the treatment of premature ejaculation: a pilot study. J Sex Marital Ther 1993, 19: 198-200 sexual function in men with premature ejaculation and controls. J Urol 1996, 156:1310-5 
53 Strassberg, DS, de Gouveia Brazão, CA, Rowland, DL, Tan, P, Slob, AK. Clomipramine in the treatment of rapid (premature) ejaculation. J Sex Marital Ther 1999, 25: 89-101

54 Chia, SJ. Management of premature ejaculation - a comparison of treatment outcome in patients with and without erectile dysfunction. Int J Androl 2002, 25: 301-5

55 Balon, B. Antidepressants in the treatment of premature ejaculation. J Sex Marital Ther 1996, 22: 85-96

56 Kotera, J, Fujishige, K, Omori, K. Immunohistochemical localization of cGMP-specific phosphodiesterase (PDE5) in rat tissues. J Histochem Cytochem 2000, 48: 685-93

57 Pfaus, JG. Neurobiology of sexual behavior. Curr Opin Neurobiol 1999, 9: 751-58

58 Hellstrom, W, Gittelamn, M, Althoff, S et al. Dapoxetine HCl for the treatment of premature ejaculation; a phase II, randomized, double-blind, placebocontrolled study. J Sex Med 2004, suppl. 1: 59

59 Burke, JB. Viewpoint: Once weekly fluoxetine. Drugs 2001, 15: 2229-30 
60 Wagstaaf, AJ e Goa, KL.Once-Weekly Fluoxetine. Drugs 2001, 15: 2221-28

61 Schmidt, ME; Fava, M, Robinson JM. The efficacy and safety of a new enteric-coated formulation of fluoxetine given once weekly during the continuation treatment of major depressive disorder. J Clin Psychiatry 2000, 11: 851-7

62 Schmidt, ME; Gonzales, J; Michelson, D. Sexual functioning during acute and continuation therapy with fluoxetine: a prospective assessment. Psychosomatics 2001, 2: 196 